\title{
Dynamic Hyperparameter Optimization for Bayesian Topical Trend Analysis
}

\author{
Tomonari Masada \\ Nagasaki University \\ 1-14 Bunkyo-machi \\ Nagasaki, Japan \\ masada@cis.nagasaki- \\ u.ac.jp \\ Tsuyoshi Hamada \\ Nagasaki University \\ 1-14 Bunkyo-machi \\ Nagasaki, Japan \\ hamada@cis.nagasaki-u.ac.jp
}

\author{
Daiji Fukagawa \\ National Institute of \\ Informatics \\ 2-1-2 Hitotsubashi \\ Chiyoda-ku, Tokyo \\ daiji@nii.ac.jp \\ Yuichiro Shibata \\ Nagasaki University \\ 1-14 Bunkyo-machi \\ Nagasaki, Japan \\ shibata@cis.nagasaki- \\ u.ac.jp
}

\author{
Atsuhiro Takasu \\ National Institute of \\ Informatics \\ 2-1-2 Hitotsubashi \\ Chiyoda-ku, Tokyo \\ takasu@nii.ac.jp \\ Kiyoshi Oguri \\ Nagasaki University \\ 1-14 Bunkyo-machi \\ Nagasaki, Japan \\ oguri@cis.nagasaki- \\ u.ac.jp
}

\begin{abstract}
This paper presents a new Bayesian topical trend analysis. We regard the parameters of topic Dirichlet priors in latent Dirichlet allocation as a function of document timestamps and optimize the parameters by a gradient-based algorithm. Since our method gives similar hyperparameters to the documents having similar timestamps, topic assignment in collapsed Gibbs sampling is affected by timestamp similarities. We compute TFIDF-based document similarities by using a result of collapsed Gibbs sampling and evaluate our proposal by link detection task of Topic Detection and Tracking.

Categories and Subject Descriptors: I.2.6 [Artificial Intelligence]: Learning; H.3.3 [Information Storage and Retrieval]: Information Search and Retrieval

General Terms: Algorithms, Experimentation

Keywords: Temporal Analysis, Topic Modeling, Topic Detection

\section{INTRODUCTION}

Bayesian approach is an outstanding trend in data mining. Especially, latent Dirichlet allocation (LDA) [4] leads to challenges in various fields $[5][7][8][14]$. In this paper, we focus on topical trend analysis and propose a new approach, called Latent dYNnamically-parameterized Dirichlet Allocation (LYNDA), by regarding the parameters of topic Dirichlet priors in LDA as a function of document timestamps. In LYNDA, a topic multinomial for each document is drawn from a Dirichlet prior whose parameters are an instantiation of a per-topic function defined over
\end{abstract}

Permission to make digital or hard copies of all or part of this work for personal or classroom use is granted without fee provided that copies are not made or distributed for profit or commercial advantage and that copies bear this notice and the full citation on the first page. To copy otherwise, to republish, to post on servers or to redistribute to lists, requires prior specific permission and/or a fee.

CIKM'09, November 2-6, 2009, Hong Kong, China.

Copyright 2009 ACM 978-1-60558-512-3/09/11 ...\$10.00. document timestamps. However, if topic Dirichlet priors are markedly different among documents, we cannot fully take advantage of Bayesian approach, because too different per-document topic multinomials result in overfitting to word co-occurrence patterns local to each document. Therefore, we regard the parameters of topic Dirichlet priors as a smooth function of continuous timestamps. Consequently, documents having similar timestamps are generated based on similar Dirichlet priors and exhibit similar topic mixture.

LYNDA has an advantage in its simplicity. We can use collapsed Gibbs sampling (CGS) for LDA [6] as is and can estimate hyperparameters in the course of CGS by using an existing gradient-based method such as L-BFGS [11] [9].

CGS for LDA provides a topic assignment to all word tokens and induces predictive word probabilities conditional on each document [16]. Since document similarities can be computed based on these probabilities, we compare LYNDA with competing methods by link detection task of Topic Detection and Tracking (TDT), where better document similarity leads to a better result. Note that our approach may be applied to other probabilistic models where Dirichlet priors are prepared for timestamped data.

\section{PREVIOUS WORKS, OUR PROPOSAL}

In this paper, we focus on probabilistic topical trend analysis. Dynamic Topic Models (DTM) [3] and its continuous time version (cDTM) [13] utilize transitions of the parameters of per-topic word multinomials for modeling document temporality, where the vectors drawn from time-dependent Gaussians are used to obtain multinomial parameters. However, Gaussian is not a conjugate to multinomial. Therefore, the authors of [10] discuss that inference becomes complicated and propose Multiscale Topic Tomography Models (MTTM) based on a completely different idea. In MTTM, the time interval is segmented into two pieces recursively to obtain a binary tree representing the inclusion among subintervals. Further, each leaf node is associated with a Poisson distribution for word generation. However, MTTM cannot be applied to continuous timestamps. When compared with these works, LYNDA is remarkable in two features below. 
First, inference is easy to implement. While LYNDA requires additional computations for hyperparameter estimation, we can use CGS for LDA as is and can adopt an existing gradient-based method for hyperparameter estimation. In contrast, the above works require heavily customised implementation. Second, LYNDA can be applied to continuous timestamps. While cDTM has this feature, a special implementation technique is required for efficient memory usage.

More appropriately, we can compare LYNDA with Topics over Time (TOT) [15], because TOT shares both above features. TOT extends LDA just by adding per-topic Beta distributions, which are defined over continuous timestamps.

However, these Beta distributions cause the following problem. The full conditional probability that topic $k$ is assigned to $i$ th token of document $j$ in TOT is proportional to $\frac{\left(n_{j k}^{\neg j i}+\alpha_{k}\right)\left(n_{k w}^{\neg j i}+\beta\right)}{\sum_{w=1}^{W}\left(n_{k w}^{\neg i}+\beta\right)} \times \frac{\Gamma\left(a_{k}+b_{k}\right)}{\Gamma\left(a_{k}\right) \Gamma\left(b_{k}\right)}\left\{\tau_{j}^{a_{k}-1}\left(1-\tau_{j}\right)^{b_{k}-1}\right\}$, where $\Gamma(\cdot)$ denotes the standard gamma function, and $\tau_{j}$ is the timestamp of document $j$. The first half corresponds to the full conditional in LDA [6]. The latter corresponds to the likelihood of Beta distribution and means that the same topic is likely to be assigned to the word tokens appearing in the documents having similar timestamps. Our preliminary experiments reveal that this likelihood grows unboundedly as CGS proceeds, and that the first half comes to play only a marginal role. To solve this problem, we should keep the likelihood comparable with the first half. In our evaluation experiment, we multiply all Beta parameters $a_{k}, b_{k}$ by the same constant and keep them less than a specific limit.

In contrast, LYNDA controls time-dependency of topic assignment not by augmenting LDA with probability distributions without priors, but by making hyperparameters time-dependent to achieve a moderate fit to timestamp data. In LYNDA, we make topic Dirichlet priors time-dependent by defining $p\left(\theta_{j} \mid f_{1}, \ldots, f_{K}\right) \propto \prod_{k} \theta_{j k}^{f_{k}\left(\tau_{j}\right)-1}$ for document $j$, where $f_{k}(\cdot)$ are per-topic differentiable functions defined over continuous timestamps. In this paper, we adopt unnormalized Gaussian density and define $f_{k}(\tau) \equiv \zeta_{k} \exp \{-(\tau-$ $\left.\left.m_{k}\right)^{2} /\left(2 s_{k}^{2}\right)\right\}$. We think that Gaussian density is appropriate to represent the dynamism of topical trends. Consequently, each topic Dirichlet prior is determined by the three parameters. $m_{k}$ specifies the position of the peak popularity of topic $k, s_{k}$ tells how wide the popularity stretches, and $\zeta_{k}$ represents the intensity of popularity. We introduce $\zeta_{k}$ to automatically rescale the unnormalized Gaussian density separately for each topic $k$. These parameters are estimated by empirical Bayes method. The log likelihood of a topic assignment can be obtained as $\log \left[\prod_{j} \frac{\Gamma\left(\sum_{k} f_{k}\left(\tau_{j}\right)\right)}{\prod_{k} \Gamma\left(f_{k}\left(\tau_{j}\right)\right)}\right.$ $\left.\frac{\prod_{k} \Gamma\left(n_{j k}+f_{k}\left(\tau_{j}\right)\right)}{\Gamma\left(\sum_{k}\left(n_{j k}+f_{k}\left(\tau_{j}\right)\right)\right)}\right]$. We maximize this by optimizing $\zeta_{k}, m_{k}$, and $s_{k}$ with L-BFGS [11][9]. However, in the preliminary experiments, $m_{k}$ showed no significant change after optimization. Therefore, we fix $m_{k}$ to $(k-1) /(K-1)$ by normalizing the timestamp domain to $[0,1]$. As a result, we can put an equal interval between the peaks of neighboring Gaussians and thus can cover the entire time interval impartially.

We compare LYNDA also with LDA where hyperparameters are optimized straightforwardly. The log likelihood of a topic assignment in LDA is obtained as $\log \left[\prod_{j} \frac{\Gamma\left(\sum_{k} \alpha_{k}\right)}{\prod_{k} \Gamma\left(\alpha_{k}\right)}\right.$ $\left.\frac{\prod_{k} \Gamma\left(n_{j k}+\alpha_{k}\right)}{\Gamma\left(\sum_{k}\left(n_{j k}+\alpha_{k}\right)\right)}\right]$. Therefore, we can determine $\alpha_{k}$ by maximizing the log likelihood. LDA with this straightforward hyperparameter estimation is denoted by HypLDA.

\section{EXPERIMENTS}

We use TDT4 dataset [1] for our link detection evaluation. This dataset is accompanied with the on-topic document sets for 40 topics of TDT 2002 competition and those for 40 topics of TDT 2003 competition. From now, we say "TDTtopic" to indicate the topics prepared for these TDT competitions. While an off-topic document set is also prepared for each TDT-topic, we regard all documents other than ontopic documents as off-topic to make evaluation reliable by using as many documents as possible. The entire dataset is used both to train probabilistic models and to compute document similarities. A similar strategy is taken when LDAbased models are evaluated in ad-hoc retrieval tasks [16]. If we split the dataset into a training and a test sets, we will face a difficulty in constructing new on-topic document sets based on this split, because distributions of on-topic documents along time axis may be heavily modified. The dataset consists of $J=96,259$ documents, $W=196,131$ unique unstemmed words, and 17,638,946 word tokens after removing stop words. We use the dates as document timestamps.

Our baseline method is TFIDF, because it is widely known that TFIDF is effective in TDT tasks [2][12]. We define the weight of word $w$ in document $j$ as $n_{j w}\left\{\log \left(J / J_{w}\right)+\right.$ $\left.\sigma \log \left(n_{j w} / n_{j}\right)\right\}$, where $J_{w}$ is the document frequency of word $w, n_{j w}$ is the term frequency of word $w$ in document $j$, and $n_{j}$ is the length of document $j$. When $\sigma=0$, we obtain the conventional TFIDF. While our weighting schema looks ad-hoc, only this could give results better than the conventional TFIDF. We set $\sigma=0.4$ and regard this as our baseline TFIDF, because other values give comparable or weaker results. We adopt cosine measure for document similarity.

When we use a result of CGS, the above weighting schema is modified as $n_{j w}\left\{\log \left(J / J_{w}\right)+\rho \log p(w \mid j)+\sigma \log \left(n_{j w} / n_{j}\right)\right\}$, where $p(w \mid j)$ denotes the predictive probability of word $w$ given document $j$. Based on preliminary experiments, we set $\rho=0.2$ and $\sigma=0.2$. For LDA and HypLDA, we have $p(w \mid j)$ $=\sum_{k} \frac{n_{j k}+\alpha_{k}}{n_{j}+\sum_{k^{\prime}} \alpha_{k^{\prime}}} \frac{n_{k w}+\beta}{\sum_{w}\left(n_{k w}+\beta\right)}$. For TOT, we have $p(w \mid j)$ $\propto \sum_{k} \frac{n_{j k}+\alpha_{k}}{n_{j}+\sum_{k^{\prime}} \alpha_{k^{\prime}}} \frac{n_{k w}+\beta}{\sum_{w}\left(n_{k w}+\beta\right)} \frac{\Gamma\left(a_{k}+b_{k}\right)}{\Gamma\left(a_{k}\right) \Gamma\left(b_{k}\right)}\left\{\tau_{j}^{a_{k}-1}\left(1-\tau_{j}\right)^{b_{k}-1}\right\}$, where a normalization is required. In Section 2, we discuss that TOT suffers unbounded increase of the likelihood of per-topic Beta distributions. Therefore, for all Beta parameters $a_{k}, b_{k}$, we put two types of limits: $a_{k}, b_{k} \leq 2$ and $a_{k}, b_{k} \leq 5$. Correspondingly, we present evaluation results under the tags TOT2 and TOT5. Since the peak of Beta density is higher for larger parameters, TOT5 is more affected by timestamp data. We use these two limits, because smaller limits make TOT indistinguishable from LDA, and larger limits heavily degrade the overall performance. Finally, for LYNDA, $p(w \mid j)=\sum_{k} \frac{n_{j k}+f_{k}\left(\tau_{j}\right)}{n_{j}+\sum_{k^{\prime}} f_{k^{\prime}}\left(\tau_{j}\right)} \frac{n_{k w}+\beta}{\sum_{w}\left(n_{k w}+\beta\right)}$.

We compare LYNDA with five methods: TFIDF, LDA with fixed hyperparameters $\left(\alpha_{k}=0.5\right.$ for all $\left.k, \beta=0.01\right)$, HypLDA, TOT2, and TOT5. The number of topics $K$ is set to 100 . While we tested $K=50$ and 200 , we only obtained worse results for $K=50$ and comparable results for $K=200$. We prepare 30 results of CGS from different random initializations. The number of iterations of CGS is 500 for LDA, TOT2, and TOT5. In the course of CGS for HypLDA and LYNDA, we estimate hyperparameters by LBFGS once for every 10 iterations. HypLDA and LYNDA require 1,000 iterations, because convergence is slow due to the incorporation of hyperparameter estimation.

Our evaluation is conducted as follows. Assume that the 


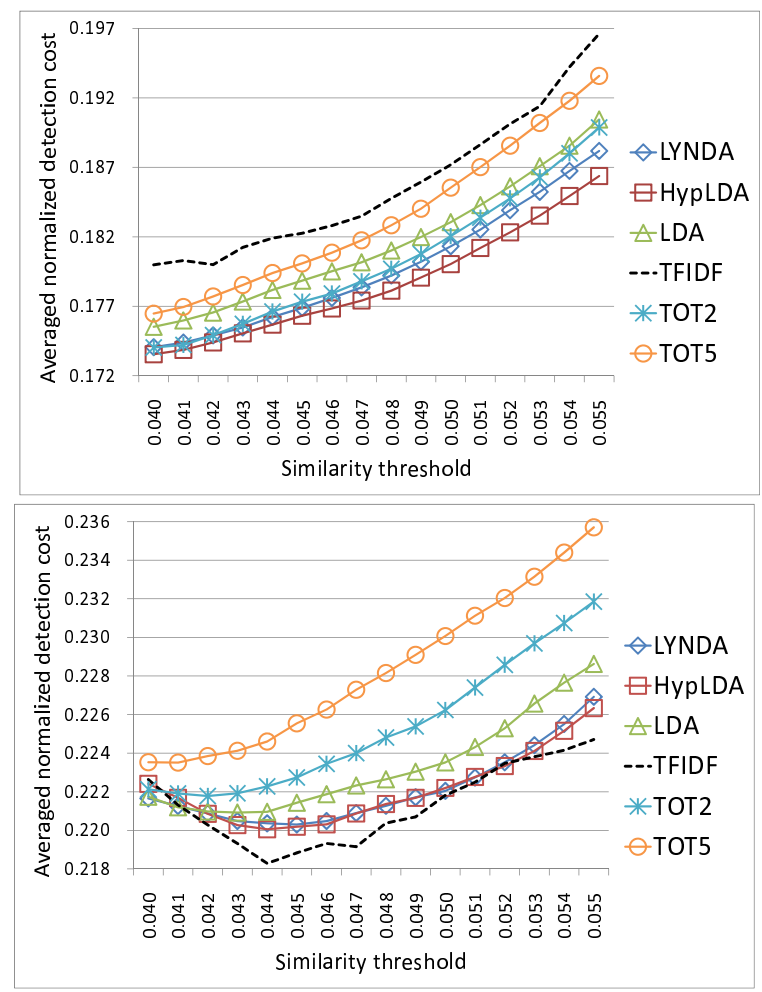

Figure 1: Comparing averaged NDCs.

number of on-topic documents for a TDT-topic $t$ is $T_{t}$. By computing the similarities between each on-topic document and all documents, we have similarities for $T_{t} \times J$ document pairs. Among these pairs, we call $T_{t} \times T_{t}$ pairs of two on-topic documents "correct" and the rest "incorrect." We would like to approximate this ideal split by devising a document similarity and then by setting a similarity threshold to obtain a split. We introduce two functions of similarity threshold $\eta . R_{t}(\eta)$ is the number of correct pairs whose similarities are larger than $\eta . A_{t}(\eta)$ is the number of incorrect pairs whose similarities are larger than $\eta$. Now we can define two evaluation measures: miss probability $P_{t}^{\text {Miss }}(\eta)$ $\equiv 1-R_{t}(\eta) /\left(T_{t} \times T_{t}\right)$ and false alarms probability $P_{t}^{F A}(\eta)$ $\equiv A_{t}(\eta) /\left\{T_{t} \times\left(J-T_{t}\right)\right\}$. We also use Normalized Detection Cost (NDC) defined as $P_{t}^{\text {Miss }}(\eta)+4.9 \times P_{t}^{F A}(\eta)$ based on an intuition that false alarms are more unfavorable [2][12].

\section{RESULTS}

Figure 1 presents NDCs for the similarity thresholds from 0.040 to 0.055 with 0.001 step. The top panel gives NDCs averaged over 40 TDT-topics of TDT 2002, and further averaged over 30 results of CGS except for TFIDF. The bottom panel gives the results for TDT 2003. Based on Figure 1, we choose $\eta=0.04$, because smaller values are advantageous to TDT 2002, and larger values are to TDT 2003. Figure 1 shows that TFIDF is efficient for TDT 2003. A detailed inspection reveals that TFIDF is likely to work for the TDT-topics whose topic explication includes characteristic words, e.g. for TDT-topic 41016 having an explication where the words "Basque" and "ETA" appear, and for 41021 whose explication includes the words "Ghana" and "Kufuor."

Based on Figure 1, we can conclude that hyperparameter estimation improves LDA, because both LYNDA and Hy-

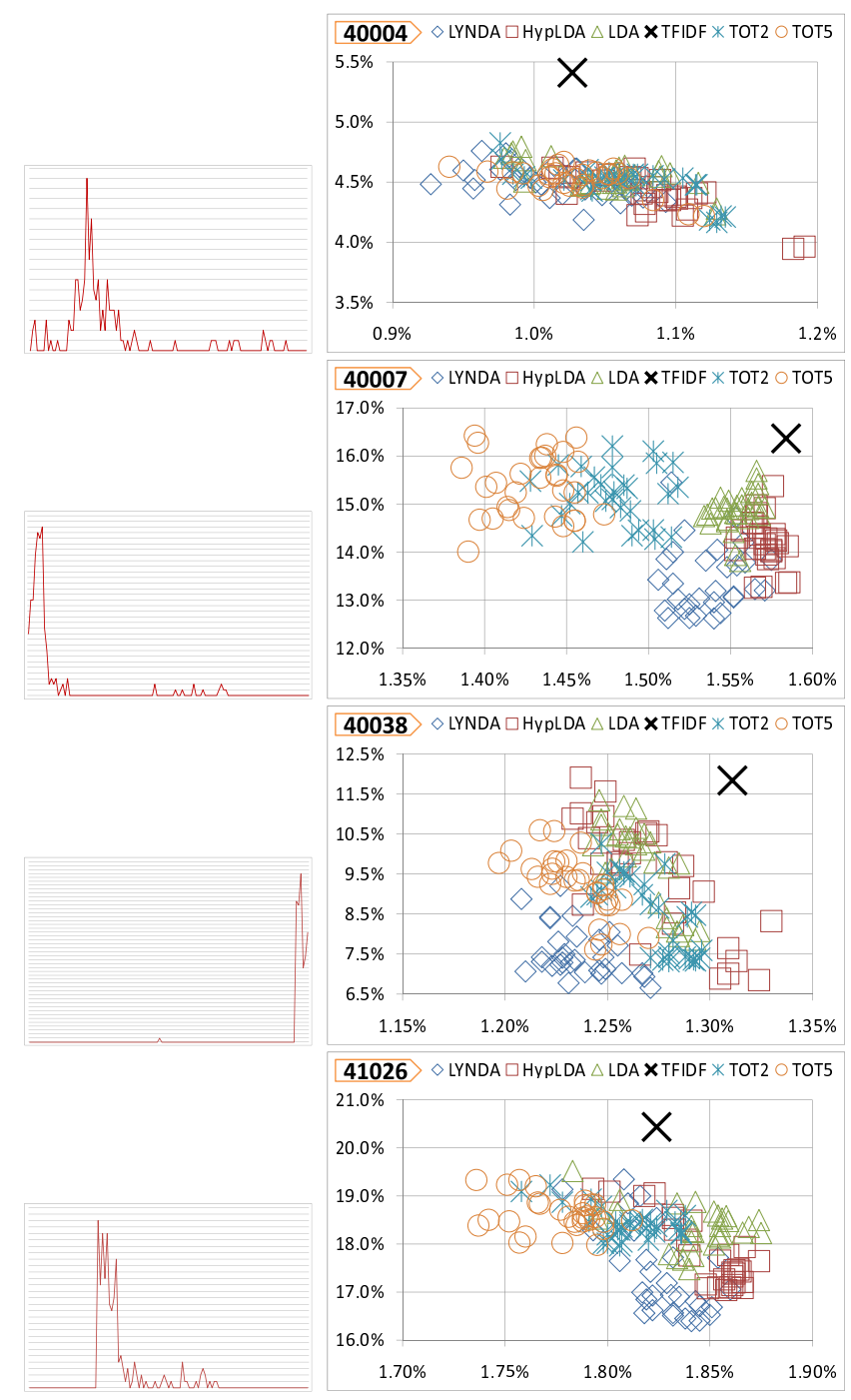

Figure 2: TDT-topics where our approach succeeds.

pLDA work better than LDA. However, there seem no significant differences between LYNDA and HypLDA. The differences will later be revealed by inspecting several TDT-topics separately. Further, Figure 1 shows that TOT results in a weak performance. However, if we inspect TDT-topics separately, we will know that TOT is comparable with LYNDA under a specific condition, and nevertheless that LYNDA is more robust with respect to overfitting to timestamp data.

In each of Figures 2 and 3, we select four TDT-topics and clarify detailed differences. Figure 2 presents the results for the TDT-topics where LYNDA succeeds, and Figure 3 for the TDT-topics where LYNDA fails. Each scatter graph is tagged with a TDT-topic ID and includes markers each of which corresponds to a pair of a false alarms probability (horizontal axis) and a miss probability (vertical axis). One cross marker is given for TFIDF, and 30 markers are for other methods, because we have 30 results of CGS except for TFIDF. The line graph on the left side of each scatter graph shows the number of on-topic documents at each date ranging from December 1 in 2000 to January 31 in 2001, where horizontal grids are put at unit interval.

First, we compare LYNDA with HypLDA and LDA. Fig- 
ures 2 and 3 provide the following observations. In these figures, we give the TDT-topics whose on-topic documents exhibit a distribution with one distinguished peak along time axis. However, smaller peaks are also observed. When the height of smaller peaks is far less than that of the largest peak, LYNDA succeeds (e.g. 40007 and 41026 in Figure 2). However, even when the height of smaller peaks is considerably less than that of the largest peak, LYNDA fails as long as some smaller peaks are placed far from the largest peak (e.g. 40026 and 41025 in Figure 3). When the height of smaller peaks is comparable with that of the largest peak, LYNDA fails (e.g. 40003 and 41014 in Figure 3). However, even when the height of smaller peaks is comparable with that of the largest peak, LYNDA succeeds as long as smaller peaks are located near the largest peak (e.g. 40004 and 40038 in Figure 2). Based on these observations, we can draw a claim: For a pair of documents having similar timestamps, LYNDA works.

Second, to compare LYNDA with TOT2 and TOT5, we observe from Figure 2 that TOT achieves the results better than or comparable with LYNDA for many of the presented TDT-topics, e.g. 40007 and 41026. That is, TOT also works for a pair of documents having similar timestamps. However, recall that Figure 1 shows weaker results for TOT, where NDCs are averaged over all TDT-topics. This means that TOT gives poor results for the TDT-topics which are not included in Figure 2, i.e., the TDT-topics whose on-topic documents do not show a concentrated timestamp distribution. Therefore, we can draw another claim: For a pair of documents located far from each other along time axis, TOT is likely to give the results worse than other methods, though LYNDA can give the results at least comparable with others.

We can combine these two claims as follows: While TOT excessively favors the TDT-topics whose on-topic documents show a concentrated timestamp distribution, LYNDA receives such TDT-topics with moderate favor. In this sense, LYNDA helps us to prevent from overfitting to timestamp data.

\section{CONCLUSIONS}

In this paper, we give a new approach for using document timestamps in LDA-based document modeling. We clarify when our approach succeeds by discussing the correlation between timestamp similarities and document similarities by comparing the results of various competing methods. However, we reveal differences between the methods only with respect to link detection task. Further investigation will be required to confirm the efficiency of our approach.

\section{REFERENCES}

[1] TDT4 data set, http://projects.ldc.upenn.edu/tdt4/.

[2] J. Allan, V. Lavrenko, and R. Nallapati. UMass at TDT 2002. In Notebook Proceedings of TDT 2002 Workshop, 2003.

[3] D. M. Blei and J. D. Lafferty. Dynamic topic models. In Proceedings of ICML'06, pages 113-120, 2006.

[4] D. M. Blei, A. Y. Ng, and M. I. Jordan. Latent Dirichlet allocation. Journal of Machine Learning Research, 3:993-1022, 2003.

[5] W.-Y. Chen, D. Zhang, and E. Y. Chang. Combinational collaborative filtering for personalized community recommendation. In Proceedings of KDD'08, pages 115-123, 2008.

[6] T. L. Griffiths and M. Steyvers. Finding scientific topics. Proc. Natl. Acad. Sci., 101 Suppl 1:5228-5235, 2004.

[7] T. Huỳnh, M. Fritz, and B. Schiele. Discovery of activity patterns using topic models. In Proceedings of UbiComp'08, pages 10-19, 2008.

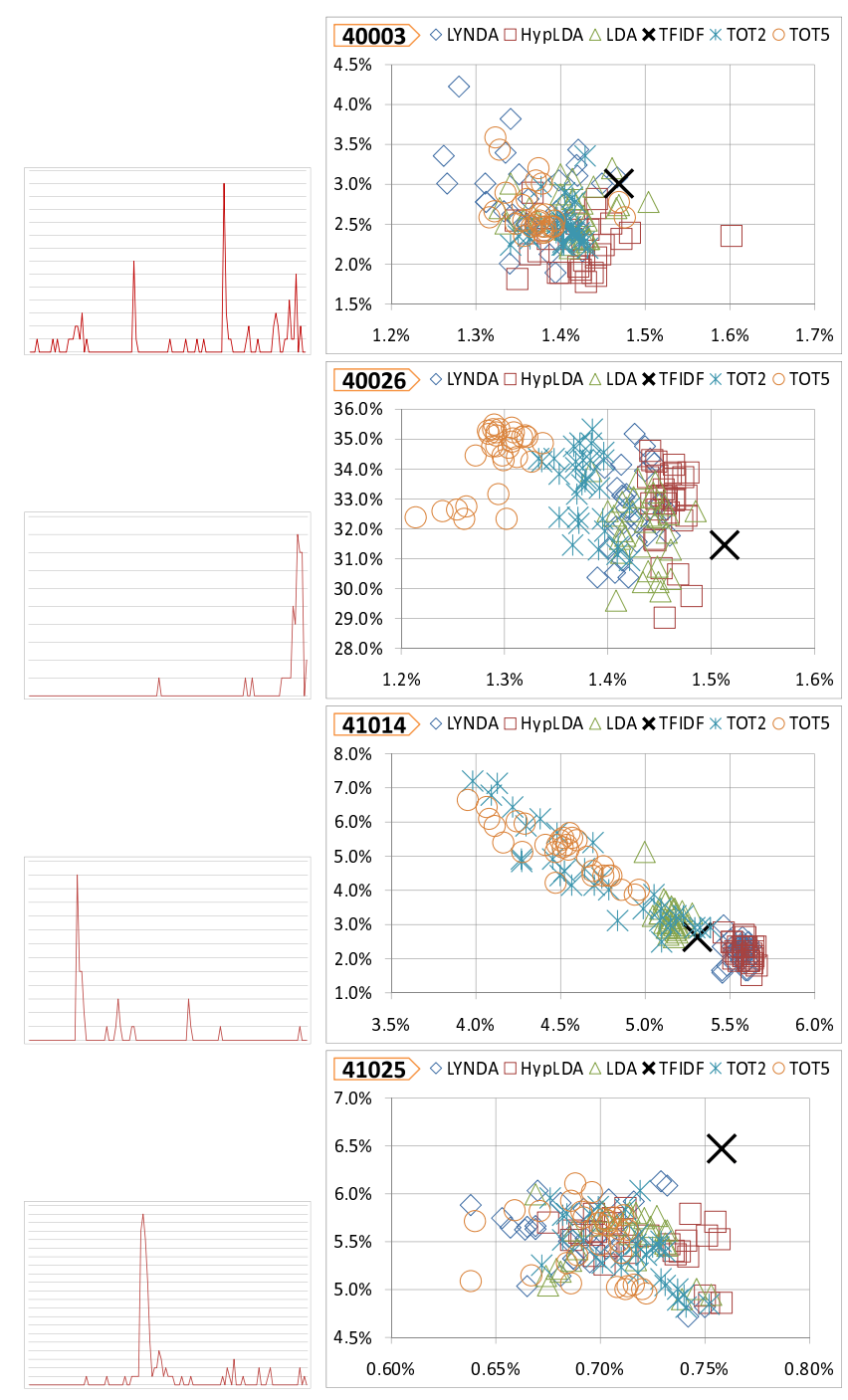

Figure 3: TDT-topics where our approach fails.

[8] E. Linstead, P. Rigor, S. Bajracharya, C. Lopes, and P. Baldi. Mining internet-scale software repositories. In NIPS 20, pages 929-936. 2008.

[9] D.-C. Liu and J. Nocedal. On the limited memory BFGS method for large scale optimization. Mathematical Programming, 45(1-3):503-528, 1989.

[10] R. M. Nallapati, S. Ditmore, J. D. Lafferty, and K. Ung. Multiscale topic tomography. In Proceedings of $K D D^{\prime} 07$, pages 520-529, 2007.

[11] J. Nocedal. Updating quasi-Newton matrices with limited storage. Mathematics of Computation, 35(151):773-782, 1980.

[12] C. Shah, W. B. Croft, and D. Jensen. Representing documents with named entities for story link detection (SLD). In Proceedings of CIKM'06, pages 868-869, 2006.

[13] C. Wang, D. Blei, and D. Heckerman. Continuous time dynamic topic models. In Proceedings of UAI'08, pages 579-586, 2008.

[14] C.-H. Wang, L. Zhang, and H.-J. Zhang. Learning to reduce the semantic gap in Web image retrieval and annotation. In Proceedings of SIGIR'08, pages 355-362, 2008.

[15] X.-R. Wang and A. McCallum. Topics over time: A non-Markov continuous-time model of topical trends. In Proceedings of KDD'06, pages 424-433, 2006.

[16] X. Wei and W. B. Croft. LDA-based document models for ad-hoc retrieval. In Proceedings of SIGIR'06, pages $178-185$, 2006. 